\title{
Charge transport in disordered semiconducting polymers driven by nuclear tunneling
}

\author{
N. J. van der Kaap, ${ }^{1}$ I. Katsouras, ${ }^{2,3}$ K. Asadi, ${ }^{2}$ P. W. M. Blom, ${ }^{2}$ L. J. A. Koster, ${ }^{1}$ and D. M. de Leeuw ${ }^{2, *}$ \\ ${ }^{1}$ Zernike Institute for Advanced Materials, University of Groningen, Nijenborgh 4, 9747 AG Groningen, The Netherlands \\ ${ }^{2}$ Max Planck Institute for Polymer Research, Ackermannweg 10, 55128 Mainz, Germany \\ ${ }^{3}$ Holst Centre, High Tech Campus 31, 5656 AE Eindhoven, The Netherlands \\ (Received 20 August 2015; revised manuscript received 4 February 2016; published 25 April 2016)
}

\begin{abstract}
The current density-voltage $(J-V)$ characteristics of hole-only diodes based on poly(2-methoxy, 5-( $2^{\prime}$ ethylhexyloxy)-p-phenylene vinylene) (MEH-PPV) were measured at a wide temperature and field range. At high electric fields the temperature dependence of the transport vanishes, and all $J-V$ sweeps converge to a power law. Nuclear tunneling theory predicts a power law at high fields that scales with the Kondo parameter. To model the $J-V$ characteristics we have performed master-equation calculations to determine the dependence of charge carrier mobility on electric field, charge carrier density, temperature, and Kondo parameter, using nuclear tunneling transfer rates. We demonstrate that nuclear tunneling, unlike other semiclassical models, provides a consistent description of the charge transport for a large bias, temperature, and carrier density range.
\end{abstract}

DOI: 10.1103/PhysRevB.93.140206

Charge transport in conjugated polymers has been under intense research, both from an experimental and theoretical point of view, in the last decades [1-3]. Due to the presence of disorder in real devices, the highly conjugated path is segmented in spatially and energetically distributed sites. As a consequence, electrical conduction in disordered conjugated polymers occurs by hopping. Baranowski et al. recently presented an overview of theories developed to describe hopping transport in amorphous inorganic and organic materials [4]. The frequency of a hopping transition $k_{i j}$ from an occupied site $i$ to an empty site $j$ is typically described either by the Miller-Abrahams hopping rate or the Marcus rate [5,6]. In both these semiclassical approaches the transition rate between the localized states is thermally activated.

From an experimental point of view, validation of such charge transport models is hindered by the fact that in organic diodes only a limited electric field and temperature range can be accessed. Due to the strong temperature dependence of the transport the device current decreases below the leakage current of the diode at temperatures typically below 100$150 \mathrm{~K}$. Furthermore, at electric fields higher than $30 \mathrm{MV} \mathrm{m}^{-1}$ electrical breakdown occurs. From a theoretical point of view the obtained parametrization for the mobility is valid only in a limited parameter space [4].

At high charge carrier density the disorder present in conjugated polymers is effectively suppressed. In recent experiments at high carrier densities using chemically highly doped in-plane diodes and ferroelectric field-effect transistors (FETs), it was demonstrated that at low temperatures there is a finite conductivity [7-11]. This is in strong contrast with the semiclassical approaches, where the conductivity is expected to vanish when the temperature approaches absolute zero. The finite conductivity at low temperatures originates from

\footnotetext{
*Corresponding author: deleeuw@mpip-mainz.mpg.de
}

Published by the American Physical Society under the terms of the Creative Commons Attribution 3.0 License. Further distribution of this work must maintain attribution to the author(s) and the published article's title, journal citation, and DOI. nuclear tunneling, which takes into account the existence of absolute-zero ground-state oscillations that drive the tunneling of the carrier between the initial and final state [12]. Based on this quantum mechanical tunneling process, Asadi et al. derived an analytical expression for the current density in the absence of disorder [12]. The authors demonstrated that the renormalized current-voltage characteristics of various polymers and devices at all temperatures collapse on a single universal curve. The analytical description of charge transport based on nuclear tunneling has been successfully applied, for instance, in studies of isotope effects on charge transport [13], hopping dynamics [14], bandlike transport [15], and transport parameters in organic semiconductors [16-18].

In another experiment, $\mu \mathrm{m}$-sized organic diodes with very small areas were used in combination with voltage pulses, which allows them to be biased at much higher electric fields (400 $\mathrm{MV} \mathrm{m}^{-1}$ ) than is possible for large-area devices [19]. It was shown that the Miller-Abrahams based mobility model only describes the current-voltage characteristics at low voltages, but is inconsistent with the data in the high voltage range [19]. At high voltages a power-law behavior was observed for the mobility as a function of electric field that could not be reproduced by theory. In the semiclassical approach of Miller-Abrahams at high electric fields, when all hops are downhill, the hopping rate is constant and the mobility decreases with increasing electric field (see Fig. 1) [5]. For the Marcus theory the transition to the inverted region will lead to mobility and current decrease at high electric field (see Fig. 1). Hence, both Marcus theory and the Miller-Abrahams formalism predict dependences of the mobility on electric field that are at variance with the experimentally observed behavior at large bias voltages [6]. What is clearly missing is a model that explains the charge transport in disordered organic semiconductors over a wide range of temperatures, carrier densities, and electric field.

In this Rapid Communication we use hopping rates based on nuclear tunneling to determine and parametrize the dependence of mobility on temperature, electric field, and charge carrier density, and use the derived expressions to describe charge transport through hole-only diodes based on poly(2-methoxy, 5-( $2^{\prime}$ ethyl-hexyloxy)- $p$-phenylene vinylene) 


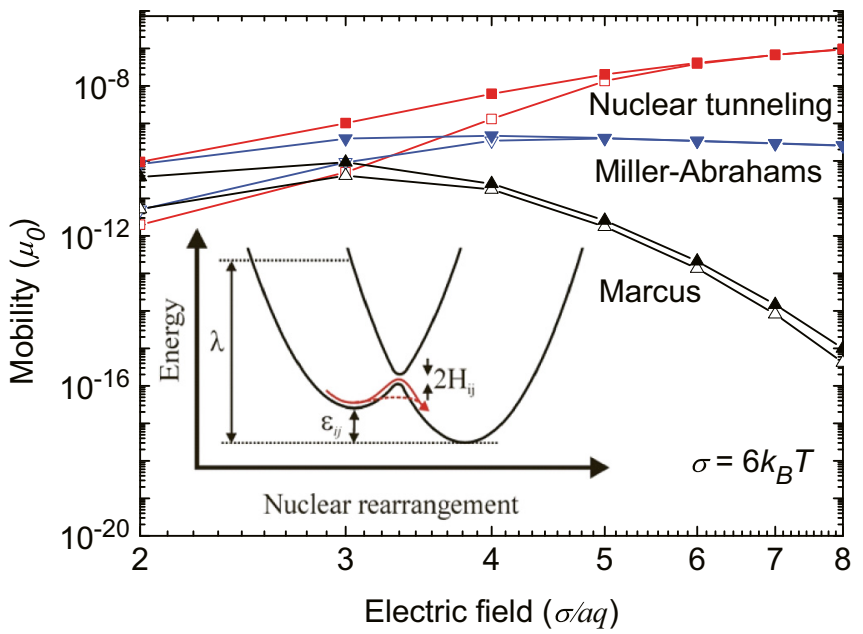

FIG. 1. The charge carrier mobility dependence for three different hopping models. Miller-Abrahams hopping and Marcus hopping both predict decreasing values for increasing fields, while nuclear tunneling hopping $\left(\alpha_{K}=2\right)$ shows an increasing mobility. The open symbols correspond to a charge density of $10^{-4} a^{-3} \mathrm{~m}^{-3}$, while the solid symbols represent data from $10^{-2} a^{-3} \mathrm{~m}^{-3}$. On the $x$ axis, the electric field is divided by the product of the lattice constant and the elementary charge. The inset represents the electron transfer in a biased double quantum well. $\epsilon_{i j}$ is the difference between the minima of the potential energy wells, $\lambda$ is the reorganization energy, and $H_{i j}$ is the tunnel splitting. The solid red arrow indicates the semiclassical Marcus hopping path while the dashed red arrow represents nuclear tunneling.

(MEH-PPV) at low charge carrier densities and high electric fields. We perform numerical master-equation calculations that yield a different charge carrier mobility behavior of nuclear tunneling hopping rates at high electric fields compared to other models (see Fig. 1). Together with the results of Asadi et al. for high carrier densities [12], the excellent fits at a wide temperature and field range in this Rapid Communication demonstrate that nuclear tunneling provides a uniform description of charge transport in organic electronic devices.

In the nuclear tunneling process the shape of the potential is determined by the coupling of the electronic charge to its nuclear environment. For the purely dissipative case, as represented by semiconducting polymers, a compact rate has been derived in the seminal work of Weiss et al. and Dorsey et al. [20,21]. In the nonadiabatic limit, this rate equation reduces to [12]

$$
\begin{aligned}
k= & \frac{H_{i j}^{2}}{\hbar^{2} \omega_{c}}\left(\frac{\hbar \omega_{c}}{2 \pi k_{B} T}\right)^{1-2 \alpha_{K}}\left|\Gamma\left(\alpha_{K}+i \frac{\epsilon_{i j}}{2 \pi k_{B} T}\right)\right|^{2} \Gamma\left(2 \alpha_{K}\right)^{-1} \\
& \times \exp \left(\frac{\epsilon_{i j}}{2 k_{B} T}\right)
\end{aligned}
$$

where $H_{i j}$ is the electronic coupling between initial and final state, $\epsilon_{i j}$ is the energy difference between donor and acceptor states, $\alpha_{K}$ is the Kondo parameter describing the coupling strength between the charge and bath, $\omega_{c}$ is the characteristic frequency of the bath, and $\Gamma$ denotes the complex gamma function. When $\hbar \omega_{c} \ll k_{B} T$, Eq. (1) will reduce to the Marcus expression.

Given nuclear tunneling transfer rates, we determine and parametrize the dependence of mobility on temperature $T$, electric field strength $F$, and charge carrier density $\rho$. As the exact coupling between hopping sites is unknown, Eq. (1) simplifies to

$$
\begin{aligned}
k_{i j} & =v_{0} \exp \left(2 \alpha a+\frac{\epsilon_{i j}}{2 k_{B} T}\right) \frac{\left|\Gamma\left(\alpha_{K}+i \frac{\epsilon_{i j}}{2 \pi k_{B} T}\right)\right|^{2}}{\Gamma\left(2 \alpha_{K}\right)}, \\
v_{0} & =\frac{1}{\hbar^{2} \omega_{c}}\left(\frac{\hbar \omega_{c}}{2 \pi k_{B} T}\right)^{1-\alpha_{K}},
\end{aligned}
$$

where $\alpha$ represents the inverse localization length assumed to be $10 a^{-1}$ [22]. The dependence of mobility on temperature $T$, electric field strength $F$, and charge carrier density $\rho$ is determined by numerically solving the master equation [23]

$$
\sum_{i \neq j} k_{i j} p_{i}\left(1-p_{j}\right)-k_{j i} p_{j}\left(1-p_{i}\right)=0,
$$

where $k_{i j}$ is the transfer rate from site $i$ to site $j$, and where $p_{i}$ denotes the occupation probability of site $i$. Subsequently, the master-equation calculations are parametrized. This parametrization is then implemented in a drift-diffusion simulation to reproduce the experimental current-voltage characteristics of disordered organic diodes.

The simulation volume consists of a simple cubic lattice with periodic boundary conditions and dimensions of at least $128 \times 128 \times 128$, where each lattice point corresponds to a hopping site. The average hopping distance $a$ equals the lattice constant. Each site has a specific energy level that contains contributions from both a Gaussian distributed density of states with a standard deviation of $\sigma$, and an electric field of $F$ that is applied in the $x$ direction. The density of states is assumed to be uncorrelated, which has been shown to better describe semiconducting polymers [24]. Moreover, for large electric fields and concentrations, the impact of disorder disappears, and the transport is not influenced by the shape of the density of states. Coulomb interactions between charge carriers are neglected, as these should only become relevant for carrier densities exceeding $10^{-2} a^{-3}$ [25]. Computational effort is reduced by limiting the transitions to the 26 nearest neighbors. At high carrier densities, this results in a small underestimate of the mobility that should not significantly affect our simulations [26]. Figure 1 shows the field dependence of the charge carrier mobility for MillerAbrahams hopping rates, Marcus hopping rates, and nuclear tunneling hopping rates that were calculated using the numerical simulation. For the semiclassical Miller-Abrahams and Marcus hopping rates, the mobility decreases with increasing electric field. In contrast, the mobility based on nuclear tunneling shows a power-law-like increase at high electric fields.

The calculations are performed for a wide range of parameters $\left(\sigma=\left[0 \ldots 10 k_{B} T\right], F=\left[0.01 \sigma a^{-1} q^{-1} \ldots 8 \sigma a^{-1} q^{-1}\right]\right.$, $\rho=\left[10^{-6} a^{-3} \ldots 10^{-1} a^{-3}\right]$, and $\left.\alpha_{K}=[2 \ldots 6]\right)$. The temperature and field dependence of the mobility for $\alpha_{K}=2$ and $F=0.01 \sigma a^{-1} q^{-1}$ is presented in Fig. 2 . The lines are fits to the mobility, following the universal mobility scaling theory, 


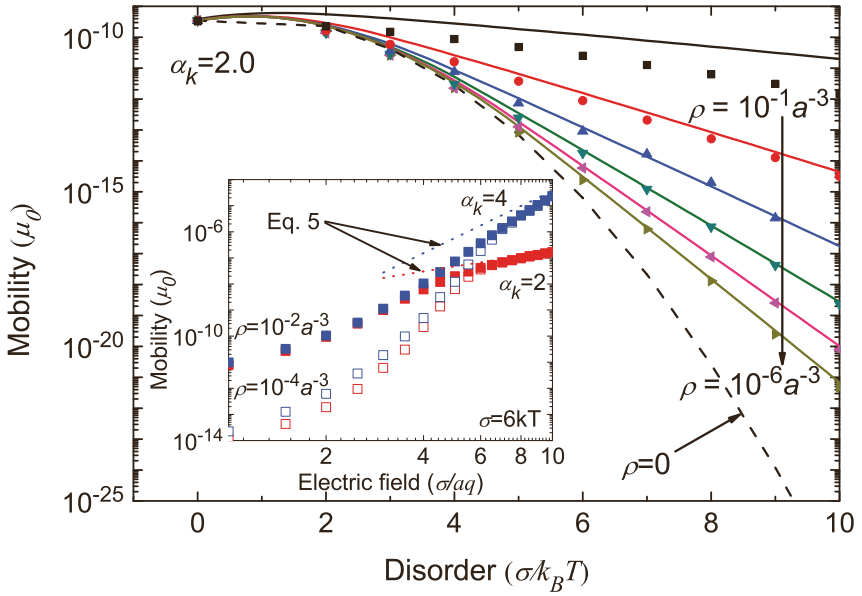

FIG. 2. Dependence of mobility on temperature and carrier density for $\alpha_{K}=2$ and $F=0.01 \sigma a^{-1} q^{-1} \mathrm{~V} \mathrm{~m}^{-1}$. The lines are given by Eq. (4). $\mu_{0}$ is given by $\frac{v_{0} a^{2} q}{\sigma}$. The inset shows the electric field dependence of the mobility for two values of $\alpha_{K}$ : For large fields, the density dependence vanishes and the mobility is given by Eq. (5).

as derived from percolation theory [27]

$$
\begin{aligned}
\mu(T) & =v_{0} \exp \left(-2 \alpha a-0.5 \sigma^{2}+0.8 \sigma\right) \frac{\left|\Gamma\left(\alpha_{K}\right)\right|^{2}}{\mid \Gamma\left(2 \alpha_{K}\right)}, \\
\mu(T, \rho) & =\frac{\mu(T)}{\rho a^{3}} \exp \left[E_{f}(T, \rho)+0.5 \sigma^{2}\right],
\end{aligned}
$$

where $E_{f}(T, \rho)$ equals the Fermi level for a certain temperature and carrier density. The master-equation results agree well with the percolation theory of Eqs. (4), except for charge carrier densities exceeding $10^{-2} a^{-3}$. The good agreement indicates that finite size effects do not occur for the range of parameters under consideration [28]. At high electric fields, the density dependence disappears, and the mobilities approach a power law that is given by

$$
\mu(T, \rho, F)=\frac{2 \pi v_{0} \exp (-2 \alpha a) \sigma}{F a \Gamma\left(2 \alpha_{K}\right)}\left|\frac{F a}{2 \pi k_{B} T}\right|^{2 \alpha_{K}-1} .
$$

The inset of Fig. 2 presents the mobility for different values of $\alpha_{K}$ and $\rho a^{3}$, where the lines correspond to Eq. (5). For clarity, mobilities for $\alpha_{K}=4$ and $\alpha_{K}=2$ have been overlapped at low electric fields, by scaling the former with a constant. At high electric fields, the mobility becomes independent of carrier density, and converges to a power law that scales with $\alpha_{K}$. Furthermore, the onset of the field range at which the mobility is described by Eq. (5) increases with increasing $\alpha_{K}$.

The hole-only diodes of Au/MEH-PPV/poly(3,4ethylenedioxythiophene):poly(styrenesulfonate)

(PEDOT:PSS) were fabricated using the molecular junction test bed, as previously described [19]. The area of the devices was defined by means of openings with a diameter ranging from 1 to $100 \mu \mathrm{m}$ in an insulating photoresist layer, by conventional UV lithography. The small area of the diodes allows them to be biased at much higher voltages than what is possible for large-area devices. The application of short voltage pulses further extends the applied electric field range [19]. Furthermore, the diodes are defined in a matrix of insulating photoresist, eliminating the problem of parasitic leakage. The current density as a function of electric field for diodes with MEH-PPV layer thicknesses of 40, 80, and $115 \mathrm{~nm}$ are presented in Fig. 3 [19]. The charge transport could be measured over an electric field range of approximately 5 decades, from 0.01 up to $300 \mathrm{MV} \mathrm{m}^{-1}$, while the temperature was varied between 175 and $300 \mathrm{~K}$ [19]. This range is much larger than in typical devices, which makes the system suitable for studying the charge transport dynamics in thin-film. The data are plotted for monopolar applied bias, but the transport characteristics were nearly symmetric for negative and positive bias, showing that the highest occupied molecular orbital (HOMO) of the MEH-PPV layer is well aligned with the Fermi level of the bottom gold contact and the HOMO of the PEDOT:PSS top electrode.

The charge transport characteristics of space charge limited (SCL) devices can be found by solving the coupled continuity and Poisson equations [29]. These equations are solved by a drift-diffusion simulation that contains a certain charge carrier mobility model [30]. The mobility model allows our simulation to mimic the behavior of disordered hopping

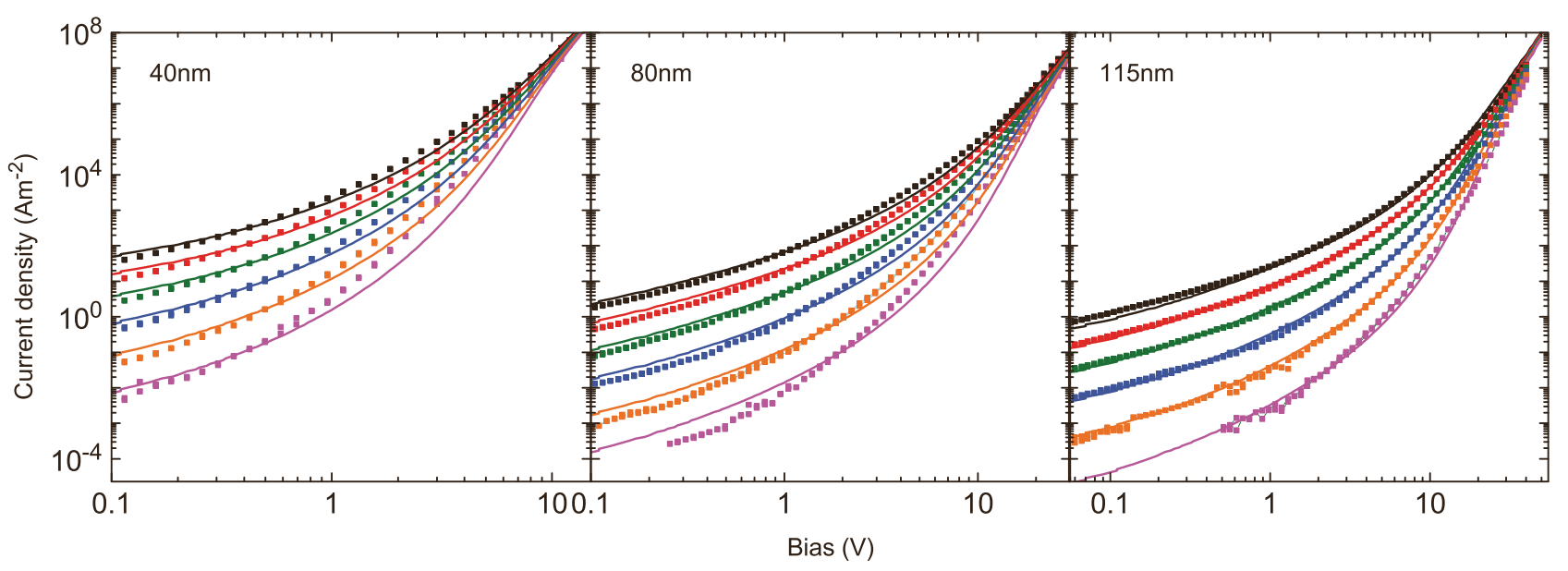

FIG. 3. Current-voltage characteristics of MEH-PPV hole-only diodes with thicknesses of 40, 80, and 115 nm measured at temperatures ranging from 175 to $300 \mathrm{~K}$. The lines correspond to drift-diffusion simulations based on a nuclear tunneling mobility model. All fits use the same values for $\alpha_{K}, \sigma, a$, and $v_{0}$. 
transport in organic semiconductors. Although the mobility can be described analytically at both low [Eq. (4)] and high electric fields [Eq. (5)], an accurate description of the intermediate field range is lacking. Therefore, instead of using an empirical relation that closely matches the master-equation results, we implement an interpolation scheme. The scheme is based on a linear interpolation algorithm, and calculates the weighted average of the master-equation results that are close to the required $F, \rho, \alpha_{K}$, and $T$. This approach avoids the introduction of fitting errors. Copies of both the interpolation code and the lookup table are included in the Supplemental Material [31].

Figure 3 shows the current-voltage characteristics of MEHPPV hole-only diodes, for a range of temperatures between 175 and $300 \mathrm{~K}$. The lines correspond to drift-diffusion simulations including the nuclear tunneling mobility model. All simulations assume an $a$ of $2.0 \mathrm{~nm}$, a $\sigma$ of $0.095 \mathrm{eV}$, and $\alpha_{K}$ of 3.0. Both contacts are Ohmic, and a small amount of ionized $p$-type doping is added with a concentration of $10^{21} \mathrm{~m}^{-3}$. For the low voltage range, the energetic disorder and hopping distance are comparable to the values that are found using Miller-Abrahams mobilities [30]. For high voltages, the measurements show that the temperature dependence in all devices disappears, and that the currents are described by Eq. (5). The good agreement between the experimental results and the simulation indicates that the devices satisfy space charge limited theory. A deviation from the cubic thickness scaling is caused by the larger charge carrier densities of thin devices [32]. Nuclear tunneling therefore, unlike MillerAbrahams and Marcus theory, can describe the experimental data throughout the wide temperature and electric field range. All data are fitted with $\alpha_{K}=3.0$, a value that is in very good agreement with experimentally obtained Kondo parameters for organic semiconductors, ranging from 1.6 to 6.75 [12].

The coupling between the heat bath and the system are described by the spectral density. For the Ohmic spectral density that is used in this work, the damping is frequency independent up to the characteristic frequency $\omega_{c}$. For frequencies larger than $\omega_{c}$, the coupling strength decays exponentially. The classical reorganization energy associated with the bath, which is twice the polaron binding energy, as schematically indicated in the inset of Fig. 1, is given by $\lambda=2 \alpha_{K} \hbar \omega_{c}$. For a typical reorganization energy of $0.6 \mathrm{eV}$, this leads to $\omega_{c}=0.1 \mathrm{eV}$. Equation (1) has been derived under the condition that $\hbar \omega_{c} \gg k_{B} T$. For the low temperature FET measurements by Asadi et al. [12], this assumption is indeed correct, but it fails for room temperature measurements. This would lead to a saturation of the current at high electric fields, which has not been observed. A possible explanation is an enhancement of the reorganization energy for high electric fields $[33,34]$. However, further investigation is required to verify this assertion.

In conclusion, we performed master-equation calculations to determine the dependence of charge carrier mobility on electric field, charge carrier density, temperature, and Kondo parameter, using nuclear tunneling transfer rates. For high electric fields, nuclear tunneling theory predicts that the mobility is expected to converge to a power law that scales with the Kondo parameter. We implemented these results in a drift-diffusion simulation to describe the current-voltage characteristics of MEH-PPV small-area hole-only diodes. For large bias, the temperature dependence vanishes, and all sweeps converge to a power law, as predicted by nuclear tunneling. We demonstrate that nuclear tunneling, unlike other semiclassical models, provides a consistent description of the charge transport for a large bias, temperature, and carrier density range. The Kondo parameter that we extracted is comparable to previously determined values, suggesting that nuclear tunneling is a generic process that describes charge transport in organic semiconductors.

The authors wish to thank M. Kuik, G. J. Wetzelaer, and H. Nicolai for fruitful discussions, J. Wildeman for the synthesis of MEH-PPV, and J. Harkema and A. Kamp for technical support. We acknowledge financial support from the Max Planck Institute for Polymer Research, the Zernike Institute for Advanced Materials, and from the European Community's Seventh Framework Programme (FP7/2007-2013) under the Grant Agreements ONE-P No. 212311. The work by N.J.v.d.K. is part of the research program of the Foundation for Fundamental Research on Matter (FOM), which is part of the Netherlands Organization for Scientific Research (NWO). K.A. acknowledges Alexander von Humboldt Foundation for the funding provided in the framework of the Sofja Kovalevskaja Award endowed by the Federal Ministry of Education and Research, Germany.

N.J.V.D.K. and I.K. contributed equally to this work.
[1] F. C. Krebs, N. Espinosa, M. Hösel, R. R. Søndergaard, and M. Jørgensen, Adv. Mater. 26, 29 (2014).

[2] H. Sirringhaus, Adv. Mater. 26, 1319 (2014).

[3] M. Kuik, G. J. A. H. Wetzelaer, H. T. Nicolai, N. I. Craciun, D. M. D. Leeuw, and P. W. M. Blom, Adv. Mater. 26, 512 (2014).

[4] A. V. Nenashev, J. O. Oelerich, and S. D. Baranovskii, J. Phys.: Condens. Matter 27, 093201 (2015).

[5] A. Miller and E. Abrahams, Phys. Rev. 120, 745 (1960).

[6] R. A. Marcus and N. Sutin, Biochim. Biophys. Acta 811, 265 (1985).
[7] J. D. Yuen, R. Menon, N. E. Coates, E. B. Namdas, S. Cho, S. T. Hannahs, D. Moses, and A. J. Heeger, Nat. Mater. 8, 572 (2009).

[8] A. J. Kronemeijer, E. H. Huisman, I. Katsouras, P. A. van Hal, T. C. T. Geuns, P. W. M. Blom, S. J. van der Molen, and D. M. de Leeuw, Phys. Rev. Lett. 105, 156604 (2010).

[9] A. S. Dhoot, G. M. Wang, D. Moses, and A. J. Heeger, Phys. Rev. Lett. 96, 246403 (2006).

[10] R. Menon, C. O. Yoon, D. Moses, A. J. Heeger, and Y. Cao, Phys. Rev. B 48, 17685 (1993).

[11] M. J. Panzer and C. D. Frisbie, Adv. Funct. Mater. 16, 1051 (2006). 
[12] K. Asadi, A. J. Kronemeijer, T. Cramer, L. J. A. Koster, P. W. M. Blom, and D. M. de Leeuw, Nat. Commun. 4, 1710 (2013).

[13] Y. Jiang, H. Geng, W. Shi, Q. Peng, X. Zheng, and Z. Shuai, J. Phys. Chem. Lett. 5, 2267 (2014).

[14] S. Jang and A. Montoya-Castillo, J. Phys. Chem. B 119, 7659 (2015).

[15] J. Lee, J. W. Chung, D. H. Kim, B. Lee, J. Parkl, S. Lee, R. Häusermann, B. Batlogg, S. Lee, I. Choi, I. W. Kim, and M. S. Kang, J. Am. Chem. Soc. 137, 7990 (2015).

[16] Z. Shuai, H. Geng, W. Xu, Y. Liao, and J. Andre, Chem. Soc. Rev. 43, 2662 (2014).

[17] G. Nan, X. Yang, L. Wang, Z. Shuai, and Y. Zhao, Phys. Rev. B 79, 115203 (2009).

[18] H. Geng, Q. Peng, L. Wang, H. Li, Y. Liao, Z. Ma, and Z. Shuai, Adv. Mater. 24, 3568 (2012).

[19] I. Katsouras, A. Najafi, K. Asadi, A. J. Kronemeijer, A. J. Oostra, L. J. A. Koster, D. M. de Leeuw, and P. W. M. Blom, Org. Electron. 14, 1591 (2013).

[20] U. Weiss, Quantum Dissipative Systems, Series in Modern Condensed Matter Physics Vol. 13 (World Scientific, Singapore, 2008).

[21] M. P. A. Fisher and A. T. Dorsey, Phys. Rev. Lett. 54, 1609 (1985).

[22] H. Bässler, Phys. Status Solidi B 175, 15 (1993).
[23] Z. G. Yu, D. L. Smith, A. Saxena, R. L. Martin, and A. R. Bishop, Phys. Rev. B 63, 085202 (2001).

[24] M. Bouhassoune, S. V. Mensfoort, P. Bobbert, and R. Coehoorn, Org. Electron. 10, 437 (2009).

[25] J. J. M. van der Holst, Ph.D. thesis, Eindhoven University of Technology, 2010.

[26] J. Cottaar and P. A. Bobbert, Phys. Rev. B 74, 115204 (2006).

[27] J. Cottaar, L. J. A. Koster, R. Coehoorn, and P. A. Bobbert, Phys. Rev. Lett. 107, 136601 (2011).

[28] S. Kim, J. Yeo, C. Im, and D. Kim, J. Korean Phys. Soc. 60, 1897 (2012).

[29] S. Selberherr, Analysis and Simulation of Semiconductor Devices (Springer, Berlin, 1984).

[30] W. F. Pasveer, J. Cottaar, C. Tanase, R. Coehoorn, P. A. Bobbert, P. W. M. Blom, D. M. de Leeuw, and M. A. J. Michels, Phys. Rev. Lett. 94, 206601 (2005).

[31] See Supplemental Material at http://link.aps.org/supplemental/ 10.1103/PhysRevB.93.140206 for Charge carrier mobility interpolating table for nuclear tunneling hopping rates.

[32] N. I. Craciun, J. J. Brondijk, and P. W. M. Blom, Phys. Rev. B 77, 035206 (2008).

[33] J. Sancho-García, G. Horowitz, J.-L. Brédas, and J. Cornil, J. Chem. Phys. 119, 12563 (2003).

[34] P. Song, Y. Li, F. Ma, T. n. Pullerits, and M. Sun, J. Phys. Chem. C 117, 15879 (2013). 